\title{
A Method for Estimating the Average Packet Error Rates of Multi-carrier Systems With Interference
}

\author{
Zaid Hijaz \\ Information and Telecommunication Technology Center \\ Department of Electrical Engineering and Computer \\ Science \\ University of Kansas \\ Lawrence, KS, USA \\ zhijaz@ku.edu
}

\author{
Victor S. Frost \\ Information and Telecommunication Technology Center \\ Department of Electrical Engineering and Computer \\ Science \\ University of Kansas \\ Lawrence, KS, USA \\ frost@ittc.ku.edu
}

\begin{abstract}
We propose a method for estimating the average packet error rates (PER) of a coded orthogonal frequency division multiplexed (OFDM) system when the signal is subject to interference with a shaped spectral density. Thus the interference has a different impact on each sub-carrier. Typically, the PER is a function of the received signal-to-noise-ratio (SNR) and the packet bits are distributed evenly amongst a fixed number of sub-carriers where each sub-carrier has the same SNR. The OFDM system under consideration here utilizes convolutional coding for error correction and $M_{n}$-ary square quadrature amplitude modulation (M-QAM) for signaling. The proposed method assumes that each sub-carrier is affected by a different interference power. The novelty in the analysis lies in equating the average interference power affecting each sub-carrier to an equivalent additive white Gaussian noise (AWGN) power. We compare PER prediction using this approach to simulation for a number of modulation and coding schemes (MCS) to validate that the proposed approximation can be used to calculate the average PER.
\end{abstract}

Keywords- Orthogonal frequency division multiplexing (OFDM), Nakagami-m fading, Interference.

\section{I.INTRODUCTION}

Significant pressure is on wireless network resources due to the explosion of data usage by mobile devices. This has led to increased frequency reuse and the advent of femtocells [16] in order to cope with demand and reduce pressure on wireless networks. Mobile ad hoc networks (MANETs) [17] and a lack of available spectrum also contribute to the potential for interference. Interference is one of the limiting factors of capacity in wireless networks.

LTE is a wireless packet based (infrastructure) network technology [8] that has become the prevalent choice in wireless networks and will continue to remain a staple for the foreseeable future. The ability to more accurately predict PER performance of multi-carrier systems could lead to better spectrum and interference management [19]. Therefore, it is of value to develop a method for calculating the average PER when a wireless link is impacted by interference with a shaped spectral density.

In [2] simulation was used to find the bit-error-rate (BER) of an uncoded multi-carrier system when subject to a fixed channel and interference. The work in [12] shows the impact a covert interferer with a shaped spectral density could potentially have on a packet-based broadband wireless network, like LTE. These systems utilize adaptive modulation and coding (AMC), hybrid auto-repeat-request (HARQ), and OFDM. The work here first finds the PER, as a function of SNR, for an OFDM system with 12 sub-carriers like a resource block (RB) in LTE. Knowledge of the PER as a function of SNR for each the MCS is a prerequisite for analyzing the impact of interference. The interaction between the target and interfering signals occurs at the physical layer and the PER analysis developed here provides the basis for performance analysis at higher layers.

Much work has been done studying the effects of interference on the error rates of single carrier systems [9], [11], and [14]. It is well documented that PER of single carrier systems are different than multi-carrier systems [15]. The work in [3-6] examines the PER of multi-carrier OFDM systems without interference. The work in [7] examines the PER of multi-carrier OFDM systems with fading but does not examine interference.

One of the challenges in finding the average PER is that the interference can impact each sub-carrier differently, i.e., each sub-carrier can have a different SNR. Therefore, the calculation of the PER in systems with coding is not straightforward due to the fact that the bits of a packet are distributed amongst multiple sub-carriers and the PER for single carrier systems is different for multi-carrier systems [14].

Closed form expressions for PERs of each MCS under consideration here are currently unavailable. Therefore, for the case without interference we utilize simulation to collect data of the PER for a set of SNRs over a RB for each MCS mode; then apply curve fitting to find a functional relationship between PERs and SNR for each MCS similar to [4]. However, here we focus on a RB of 12 sub-carriers as in LTE. It is to be noted that all sub-carriers utilize the same MCS, i.e., mode, based on channel state information (CSI). Therefore, we present results for each mode. This paper shows the average SNR over all sub-carriers in a $\mathrm{RB}$ can be used as an approximation to determine the impact of interference

This work was supported in part by the NSF under Grant CNS-1216132. 
generated by a signal with a shaped spectral density. The SNR each sub-carrier experiences is calculated first and then averaged. This average SNR is then used to analytically calculate the average PER for each MCS mode with Nakagami- $m$ fading and interference. To validate this approach, we simulate the average PER of each scheme with interference and fading and compare it to the analytic result.

The assumptions are presented in the next section. The development of the functional relationships between PERs and SNR for each MCS is presented in Section 3. Section 4 analyzes the average PERs of the MCSs when subject to fading and interference. Section 5 validates the method in Section 4. Conclusions are discussed in the last section.

\section{SYSTEM CONFIGURATION}

Adaptive mechanisms, such as AMC, allow the communications link the ability to meet a performance target, such as PER, in a changing fading environment while maximizing throughput. AMC achieves this by pairing a specific signal modulation, M-QAM in this case, with a coding rate that meets or exceeds the PER requirement. The selection of the particular MCS is based upon the SNR at the receiver. The work here considers a system similar to an LTE system. LTE specifies a 12 sub-carrier RB and has 16 AMC modes with turbo codes for error correction [8]. LTE utilizes 4, 16, and 64-QAM signaling. The proposed system considered here (Fig. 1) utilizes a 12 sub-carrier RB, 6 AMC modes, and convolutional codes in order to simplify the analysis. The work here considers coding rates of $1 / 2,9 / 16$, and $3 / 4$. The number of bits per packet is 1080 and is distributed evenly amongst the 12 sub-carriers that comprise the RB [4]. No bits are sent when the received SNR falls below the minimum SNR threshold for mode $1, \gamma<\gamma_{1}$. The convolutional encoder, with generator polynomials $\mathrm{g}_{1}=133$ and $\mathrm{g}_{2}=171$, is terminated at the zero state by appending six zeros to the uncoded packet bits. Hard decision decoding is used at the Viterbi decoder. The puncture pattern for the rate $1 / 2,9 / 16$, and $3 / 4$, respectively, are [1 1 , [ [ 11

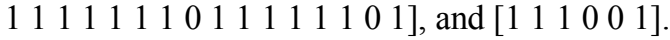

\section{SIMULATION OF PACKET ERROR RATES}

The PER, as a function of SNR, is utilized to find the expected value of the PER when the system is subject to fading and interference for each MCS. A closed form expression for the PER of each of the MCS in the proposed system is not available. Similarly, work in [7] finds the PER functions for convolutionally coded OFDM-based WLANs in fading and finds through analysis the average PER with and without fading. The work in [4] finds the PER functions of HiperLAN systems which are then utilized to find the average PER of a single carrier system when AMC and fading is considered.

Here we utilize simulation to collect data of the PER for a set of SNRs over a RB for each MCS and then curve fitting is applied to find a functional expression relating PER to SNR as in [4]. The simulation results from these simulations are then used to predict the average PER of the multi-carrier RB. The results given in Fig. 2, denoted by a star, were obtained by processing 10,000 packets at each SNR. The least squares method was utilized to fit the data to an analytic expression for each mode.

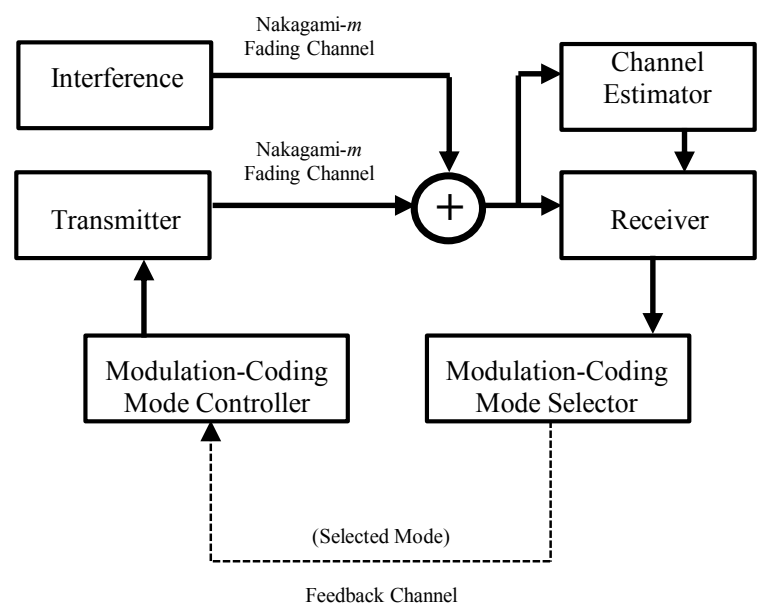

Fig. 1. System diagram with interfering transmitter.

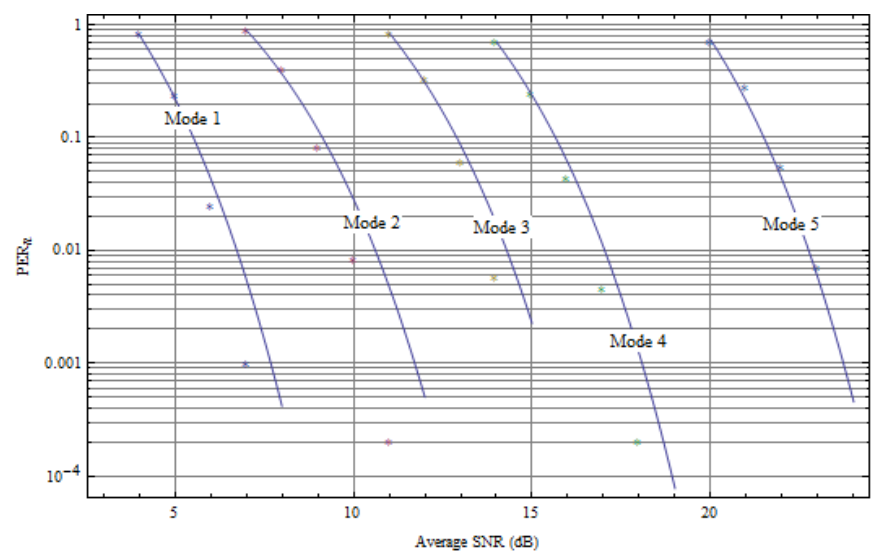

Fig. 2. Packet error rate based on simulation of MCS (stars) and curve fitting to the simulation (line). form

As in [4] and [5], the curve fit functions used here have the

$$
\operatorname{PER}_{n}(\gamma) \approx\left\{\begin{array}{c}
1, \text { if } 0<\gamma<\gamma_{p n} \\
a_{n} e^{-g_{n} \gamma}, \text { if } \gamma \geq \gamma_{p n}
\end{array}\right.
$$

where $n$ represents the mode and $\gamma$ is the received SNR. The parameters $a_{n}, g_{n}$, and $\gamma_{p n}$ are the curve fitting parameters and given in Table 1 .

\section{AVERAGE PACKET ERROR RATE WITH FADING AND INTERFERENCE}

We use the PER as a function of SNR (1) to predict the system performance when the received signal is subject to interference and fading. Both the target and interfering signals are subject to independent Nakagami- $m$ fading with the same $m$ fading parameter and flat fading is assumed. Optimum receiver structures are utilized; perfect channel state information (CSI) for the target transmitter/receiver pair and ideal phase and frequency synchronization is presumed. The interfering signal utilizes 4-QAM signaling and has the same bandwidth as a 
Table 1

\begin{tabular}{c||c|c|c|c|c}
\hline & Mode 1 & Mode 2 & Mode 3 & Mode 4 & Mode 5 \\
\hline \hline Modulation $\left(M_{n}\right)$ & QPSK & QPSK & 16 -QAM & 16-QAM & 64-QAM \\
\hline Coding Rate $R_{c}$ & $1 / 2$ & $3 / 4$ & $9 / 16$ & $3 / 4$ & $3 / 4$ \\
\hline Rate (bits/sym) & 1.00 & 1.50 & 2.25 & 3.00 & 4.50 \\
\hline$a_{n}$ & 124.9390 & 28.8989 & 42.9191 & 47.9713 & 97.5387 \\
\hline$g_{n}$ & 1.9992800 & 0.6920240 & 0.3116290 & 0.1679410 & 0.0488942 \\
\hline$\gamma_{p n}(\mathrm{~dB})$ & 3.82877 & 6.86698 & 10.81470 & 13.62620 & 19.71630 \\
\hline
\end{tabular}

target system sub-carrier. We place the interfering signal in the guard band of the target signal as in Fig. 3.

The target OFDM and interfering systems are not in synchronization. Therefore, the interfering and target signals are not orthogonal. The in-band interference is set equivalent to white Gaussian noise with a noise power equal to the in-band interference power; the main purpose of this experiment is to validate this equivalence. A similar approach was used in [9], [10], and [11] to find BER for uncoded systems. We define inband interference power as the power within the zero-to-zero crossings of the spectrum of the interfering signal, here $\sim \operatorname{sinc}^{2}(f / \mathrm{BW})$.

The pdf of the instantaneous received SNR when the target and interfering signals experience independent Nakagami- $m$ fading was developed in [14]. A form of the resulting pdf [12] is given as

$$
\begin{gathered}
f_{\gamma}(\gamma)=\frac{1}{2 \sqrt{\pi} \gamma \Gamma(m)} m^{2 m}\left(\frac{1}{\bar{\gamma}_{c}}\right)^{m}\left(\frac{\gamma}{\bar{\gamma}}\right)^{m} e^{\frac{m\left(\bar{\gamma}-\bar{\gamma}_{c} \gamma\right)}{2 \bar{\gamma} \bar{\gamma}_{c}}} \\
\left(m\left(\frac{\gamma}{\bar{\gamma}}+\frac{1}{\bar{\gamma}_{c}}\right)\right)^{\frac{1}{2}-m}\left(K_{\frac{1}{2}-m}\left(\frac{m\left(\bar{\gamma}+\bar{\gamma}_{c} \gamma\right)}{2 \bar{\gamma} \bar{\gamma}_{c}}\right)+\right. \\
\left.K_{-m-\frac{1}{2}}\left(\frac{m\left(\bar{\gamma}+\bar{\gamma}_{c} \gamma\right)}{2 \bar{\gamma} \bar{\gamma}_{c}}\right)\right),
\end{gathered}
$$

where $K_{k}$ is the modified Bessel function of the second kind [13] and $\bar{\gamma}_{I}$ is the average in-band transmitted SNR of the interfering signal. The OFDM signal is comprised of multiple carriers organized into RBs as in LTE [8] where each RB contains 12 sub-carriers. The different impact of the interfering signal on each sub-carrier in the target system must be taken into account. The influence of the interfering signal on a subcarrier decreases as the spectral distance between the interfering signal and each sub-carrier increases. To account for the different interference power on each sub-carrier, $i$, a different normalization (impact) factor is needed and can be found as in [18] by

$$
\rho_{i}=\frac{1}{B_{c}} \int_{f_{i}-\frac{\mathrm{B}}{2}}^{f_{i}+\frac{\mathrm{B}}{2}} \operatorname{sinc}^{2}\left(\pi \frac{f-k f_{s}}{B_{c}}\right) \mathrm{d} f
$$

where $f_{s}$ is the sub-carrier spacing (in this case we assume $f_{s}=$ $15 \mathrm{kHz}$ as in LTE), $B$ is the sub-carrier bandwidth, and $B_{c}$ is the bandwidth of the interfering signal. With an interfering signal power of $P_{c}$, the in-band interference for sub-carrier $i$ is $N_{i}=\rho_{i} P_{c}$, which is the contribution of the interfering signal to the average SNR per sub-carrier $i$ in the OFDM signal. We approximate the impact of the interference over the entire $\mathrm{RB}$ by using the average in-band transmitted SNR over all subcarriers in a RB and setting it equal to $\bar{\gamma}_{I}$ in equation (3), i.e.,

$$
\bar{\gamma}_{I}=P_{c} \frac{\sum_{i=1}^{I} \rho_{i}}{I}
$$

The contribution of the work here is the applications of (4) to predicting the PER over a RB and its validation. If the PER of a coded multi-carrier system without interference is known, then the impact of interference with a shaped spectral density can be found using (4).

The work here considers a slow fading channel model as in [3-6]. This means the channel does not change from the time the channel is measured until the packet is received [5]. Therefore, the average PER for a given mode in the presence of an interferer with a shaped spectral density over a RB as a function of the average transmitted target system power is defined to be

$$
\overline{\operatorname{PER}}_{n}(\bar{\gamma})=\int_{0}^{\infty} P E R_{n}(\gamma) f_{\gamma}(\gamma) d \gamma
$$

where $\bar{\gamma}_{I}$ from (4) is used in $f_{\gamma}(\gamma)$ and $P E R_{n}(\gamma)$ for each mode $n$ is from (1).

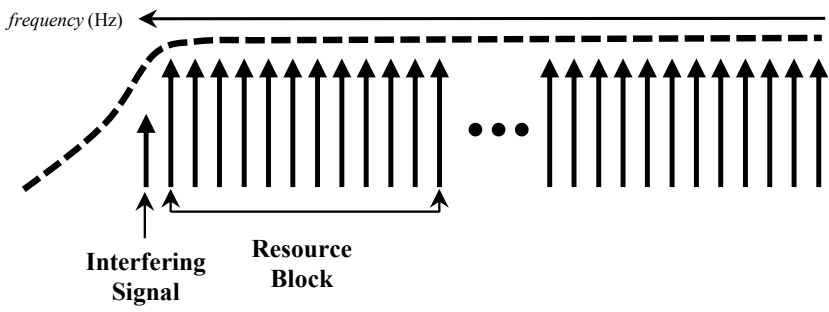

Fig. 3. Placement of the interfering signal in the guard band of the target downlink signal spectrum.

\section{VAlidation OF AVERAGe PACKET ERROR RATES}

In this section we present results that validate the analysis in the previous section. 


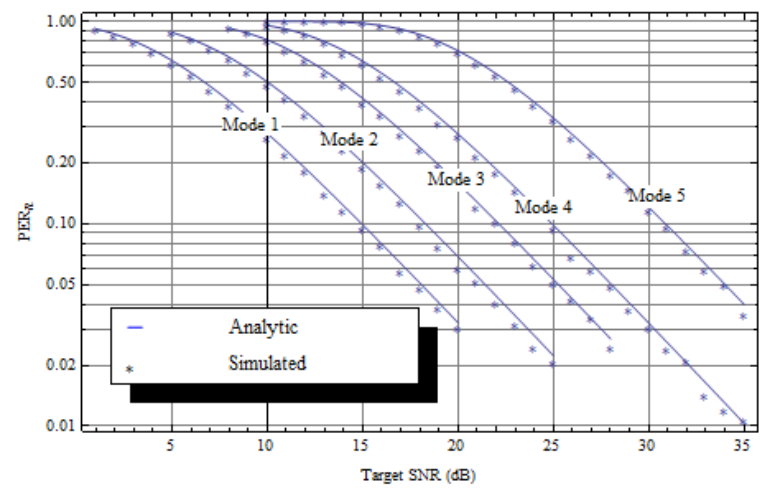

Fig. 4. Simulation of average PER (stars) and analytic prediction (line), Rayleigh fading, interfering signal $\mathrm{SNR}=5 \mathrm{~dB}$.to the simulation (line).

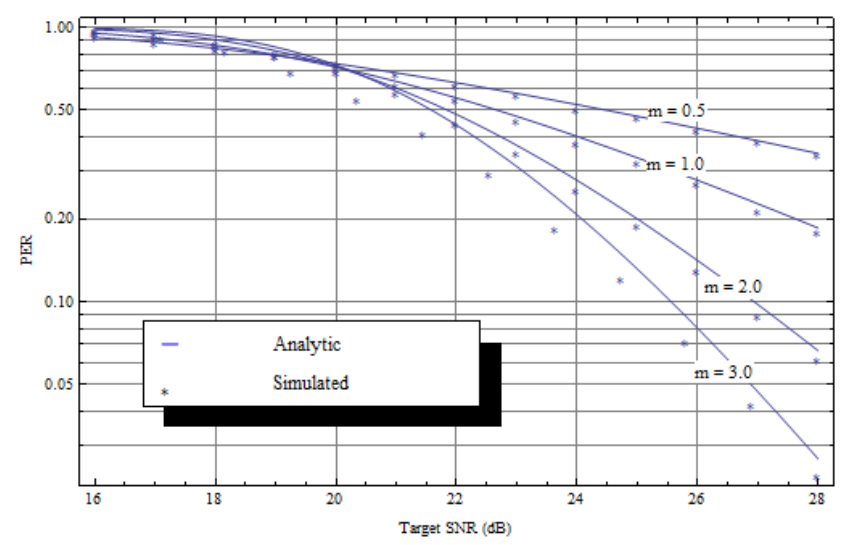

Fig. 5. Simulation of average PER (stars) and analytic prediction (line) for mode 5, Nakagami- $m$ fading, interfering signal $\mathrm{SNR}=5 \mathrm{~dB}$.

The purpose of the first experiment is to compare the average PER predicted using (4) and (5) for all the modes of the proposed system with simulation. We first set the Nakagami- $m$ fading parameter to $m=1$ (Rayleigh fading) for all modes. The interfering signal has an average transmitted SNR of $5 \mathrm{~dB}$. Fig. 4 shows that analytic curves follow the simulation closely and are more conservative than the analysis predicts.

The second experiment looks at the impact the channel has on the ability of the analysis to predict average PER performance. We select mode 5 and simulate Nakagami- $m$ fading for $m=0.5,1.0,2.0$, and 3.0 for comparison. Fig. 5 shows that the analysis performs in a fashion similar to Fig. 4; the simulated curves follow the analytic curves with only a small difference. The proposed approximation predicts worse performance for the target system than what the simulation revealed.

In the next experiment we utilize mode 1 and hold the interferer SNR steady throughout the simulation at $15 \mathrm{~dB}$. We can see from Fig. 6 that as $m$ increases the difference between the analytic prediction and the simulation increases.

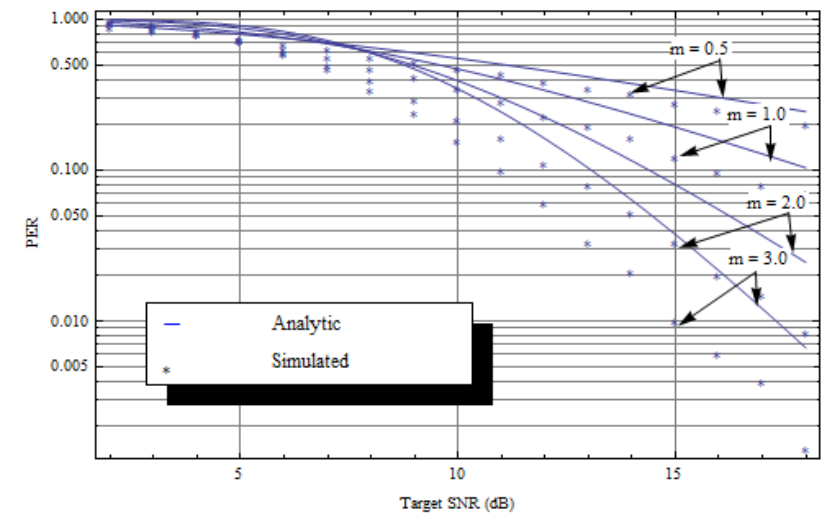

Fig. 6. Simulation of average PER (stars) and analytic prediction (line) for mode 1 , Nakagami- $m$ fading, interfering signal $\mathrm{SNR}=15 \mathrm{~dB}$.

The final experiment broadens the design space by considering other interfering signal powers. We compare the ability of the analysis to predict the target system performance when interfering signal SNR is increased from 5 to 15 and then $25 \mathrm{~dB}$ for mode 5. Fig. 7 reveals that the calculated performance of the target system is again a more conservative prediction of PER. The difference between the analytic and simulated curves increases as the interfering SNR increases; however at an interfering signal SNR of $25 \mathrm{~dB}$ the analytic prediction is off by $3 \mathrm{~dB}$.

These results show that the proposed method closely predicts the PER performance when the interference power is small. Thus in this case the impact of the interferer is mostly on the 1st sub-carrier. As the interference power increases the effect on subsequent sub-carriers increases and hence the estimate becomes less accurate however the proposed method always provides a conservative prediction of PER performance. This means that equating the in-band interference to white noise is overestimating the effect of the interference; white noise of the same power as in-band interference impacts the target signal worse than a shaped power spectrum. So the actual performance will be better than the predicted by the analysis.

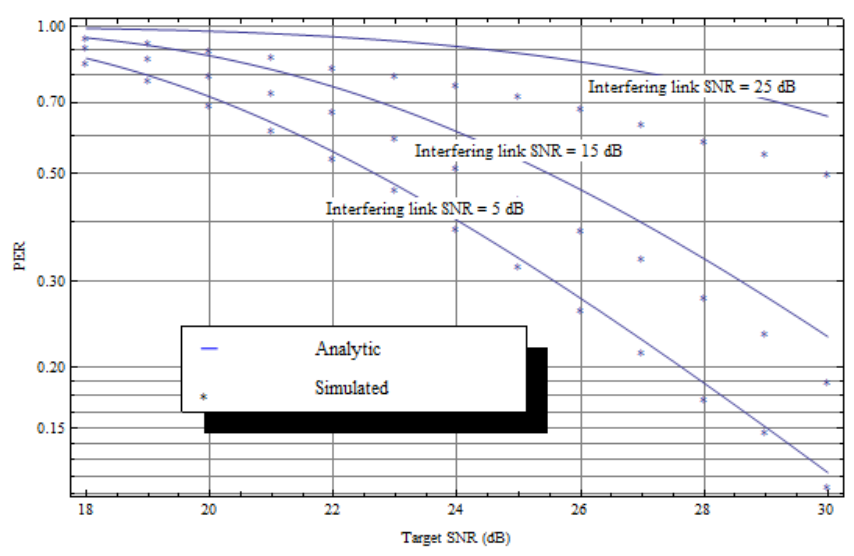

Fig. 7. Simulation of average PER (stars) and analytic prediction (line) for mode 5 with varying covert signal SNR, Rayleigh fading. 


\section{CONCLUSIONS AND FUTURE WORK}

A new method for estimating the average PER of a coded OFDM system subjected to interference with a shaped spectral density was proposed and validated. Results were presented that compared the performance of the proposed method for PER estimation to simulation. These results validated using the average in-band transmitted SNR over all sub-carriers as an approximation to determine the average PER. The method presented is a conservative predictor of performance and in all cases presented is conservative, i.e., one can expect better target system performance than predicted by the analysis. The prediction of PER performance using the proposed approach could contribute to better spectrum and interference management schemes. Also, this method can provide the basis to predict end-to-end performance including impacts on higher network layers, e.g., TCP, when a wireless hop is exposed to interference with a shaped spectral density.

\section{REFERENCES}

[1] M. Ergen, Mobile Broadband; Including WiMAX and LTE. Springer, 2009.

[2] Z. Hijaz and V. S. Frost, "Exploiting OFDM systems for covert communication," in Military Comm. Conf. 2010 - MILCOM 2010, 2010, pp. $2149-2155$

[3] X. Wang, Q.Liu, and G. B. Giannakis, "Analyzing and optimizing adaptive modulation and coding jointly with ARQ for QoS-guaranteed traffic," in IEEE Transactions on Vehicular Technology, vol. 56, pp. 710-720, 2007.

[4] Q. Liu, S. Zhou, and G. B. Giannakis, "Cross-layer combining of adaptive modulation and coding with truncated ARQ over wireless links," in IEEE Transactions on Wireless Communications, vol. 3, pp. 1746-1755, 2004

[5] Q. Liu, S. Zhou, and G. B. Giannakis, "Queuing with adaptive modulation and coding over wireless links: cross-layer analysis and design," in IEEE Transactions on Wireless Communications, vol. 4, pp. 1142-1153, 2005.

[6] Q. Liu, S. Zhou, and G. B. Giannakis, "TCP performance in wireless access with adaptive modulation and coding," in IEEE International Conference on Communications, 2004, pp. 3989-3993, Vol.7.
[7] O. Awoniyi and F. Tobagi, "Packet error rate in OFDM-Based wireless LANs operating in frequency selective channels", in INFOCOM 2006.

[8] Evolved Universal Terrestrial Radio Access (E-UTRA); Physical channels and modulation, 3GPP TS 36.211, September 2012.

[9] L. Jun, J. H. Andrian, and Z. Chi, "Bit Error Rate Analysis of jamming for OFDM systems," in Wireless Telecommunications Symposium, 2007. WTS 2007, 2007, pp. 1-8.

[10] M. K. Simon and M. S. Alouini, Digital communication over fading channels. Wiley-IEEE Press, 2004

[11] K. Hamid, "A Useful Technique for Interference Analysis in Nakagami Fading", in IEEE Transactions on Communications, Volume 55, Issue 6, 2007.

[12] Z. Hijaz, V. Frost, "The impact of interference from a covert link on a Data Link using OFDM, AMC, and Hybrid ARQ", in IEEE IPCCC 2014.

[13] C. R. Wylie, Advanced Enginering Mathematics. New York, New York: McGraw Hill, 1966.

[14] V. A. Aalo, J. Zhang, "Performance Analysis of Maximal Ratio Combining in the Presence of Multiple Equal-Power Cochannel Interferers in a Nakagami Fading Channel", in IEEE Trans. on Veh. Technol., vol. 50, no. 2, March 2001.

[15] Z. Wang, X. Ma, and G. Giannakis, "OFDM or single carrier block transmission", in IEEE Trans. on Comm., vol. 52, no. 3, March 2004.

[16] A. Zyoud, M. H. Habaebi, J. Chebil, M. R. Islam, "Femtocell interference mitigation," in IEEE Control and System Grad. Research Coll., pp. 94-99, 2012.

[17] M. Bugliesi, L. Gallina, A. Marin, S. Rossi, S. Hamadou, "Interferencesensitive preorders for MANETS," in Int. Conf. on Quan. Eval. Of Systems, pp 189-198, 2012.

[18] J. Park, D. Kim, C. Kang, and D. Hong, "Effect of partial band jamming on OFDM-based WLAN in 802.11g," in IEEE International Conf. on Acoustics Speech, and Signal Proc., 2003 - (ICASSP '03) 2003, pp. IV560-3, vol. 4

[19] N. Saquib, E. Hossain, L. B. Le, and D. Kim, "Interference management in OFDMA femtocell networks: issues and approaches," in IEEE Wireless Communications, vol.19, no.3, pp.86-95, June 2012.

[20] H. Chen, H. C. Chan, C. Chan, and V. Leung, " QoS based cross-layer scheduling for wireless multimedia transmissions with adaptive modulation and coding," in IEEE Trans. on Comm., vol. 61, no. 11, November 2013. 